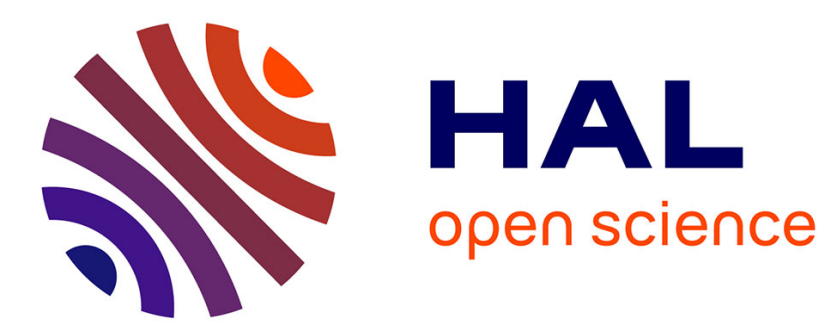

\title{
Wearable pre-impact fall detection system based on 3D accelerometer and subject's height
}

Felipe Augusto Sodré Ferreira de Sousa, Christophe Escriba, Eli Gabriel Avina Bravo, Vincent Brossa, Jean-Yves Fourniols, Carole Rossi

\section{- To cite this version:}

Felipe Augusto Sodré Ferreira de Sousa, Christophe Escriba, Eli Gabriel Avina Bravo, Vincent Brossa, Jean-Yves Fourniols, et al.. Wearable pre-impact fall detection system based on 3D accelerometer and subject's height. IEEE Sensors Journal, 2022, 22 (2), pp.1738 - 1745. 10.1109/JSEN.2021.3131037. hal-03452563

\author{
HAL Id: hal-03452563 \\ https://hal.laas.fr/hal-03452563
}

Submitted on 16 Dec 2021

HAL is a multi-disciplinary open access archive for the deposit and dissemination of scientific research documents, whether they are published or not. The documents may come from teaching and research institutions in France or abroad, or from public or private research centers.
L'archive ouverte pluridisciplinaire HAL, est destinée au dépôt et à la diffusion de documents scientifiques de niveau recherche, publiés ou non, émanant des établissements d'enseignement et de recherche français ou étrangers, des laboratoires publics ou privés. 


\begin{abstract}
This study presents a low-power wearable system able to predict a fall by detecting a pre-impact condition, performed through a simple analysis of motion data (acceleration) and height of the subject. The system can detect a fall in all directions with an average consumption of $5.91 \mathrm{~mA}$; i.e., it can monitor the activity of daily living (ADL), whether or not a fall occurs. The entire detection system uses a single wearable tri-axis accelerometer placed on the waist for the comfort of the wearer during a long-term application. The algorithm is based on the following hypothesis: "A region defined as balanced boundary circle, based on the user's height, is characterized by the fact the chance that an actual fall happening is minimal. When an activity is classified outside this circle, an acceleration analysis is performed to determine an impending fall condition". Our threshold-based algorithm was validated experimentally, first with 9 young healthy volunteers performing both normal ADL and fall activities and then using 10 ADL and 5 falls from public SisFall dataset. The results show that falls could be detected with an average lead-time of $259 \mathrm{~ms}$ before the impact occurs, with minimal false alarms (97.7\% specificity) and a sensitivity of $92.6 \%$. This is a good lead-time achieved thus far in pre-impact fall detection, permitting the integration of our detection system in a wearable inflatable airbag for hip protection.
\end{abstract}

Customizable algorithm, Fall Detection System, Pre-impact detection, Threshold-based, Wearable systems.

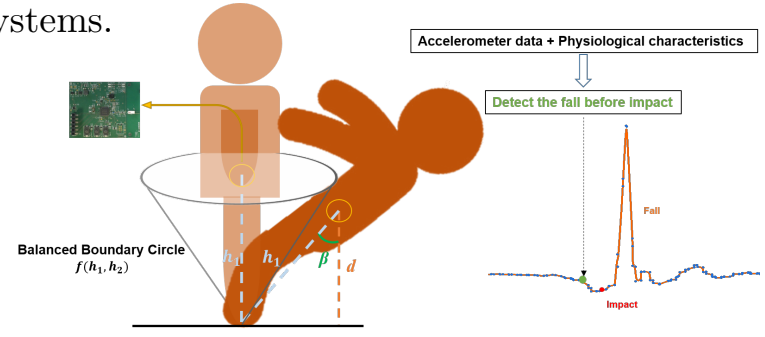




\title{
Wearable pre-impact fall detection system based on 3D accelerometer and subject's height
}

\author{
Felipe Augusto Sodré, Christophe Escriba, Eli Gabriel Avina Bravo, \\ Vincent Brossa, Jean-Yves Fourniols and Carole Rossi ${ }^{* \dagger \ddagger \S \mathbb{\|} * *}$
}

December 16, 2021

\section{Introduction}

Equipment for the protection and safety of individuals is undergoing increasing technological development. Due to the ageing process of the world population, a new research sector is gaining interest, namely, the security of the elderly. The risk of falls increases with ageing [1], and it is estimated that $30 \%$ of elderly individuals aged 65 or older fall at least once a year $[2,3]$. According to the World Health Organization, by 2050, one over six people in the world will be older than age 65. Approximately 1 out of 5 falls result in a serious injury, such as hip fracture, subdural haematoma, and other more serious injuries, including head injury, which can lead to death $[4,5]$. However, even if a fall does not result in injury, serious psychological trauma may still occur, reducing the independence and the ability to perform daily activities, such as dressing, bathing, or housekeeping; therefore, fall prevention is now recognized as an important health issue within modern countries [6]. Most of fall detection systems (FDS) [7-10] use the absence of movement,

\footnotetext{
${ }^{*}$ This work was supported by the European Research Council (H2020 Excellent Science) Researcher Award (grant 832889 - PyroSafe) and the Occitanie Region / European Union for their FEDER support (THERMIE grant).

${ }^{\dagger}$ F. A. SODRE is with University of Toulouse, Laboratory for Analysis and Architecture of Systems, LAAS-CNRS, F-31077, Toulouse, France (fasodrefer@laas.fr).

${ }^{\ddagger}$ C. ESCRIBA is with University of Toulouse, Laboratory for Analysis and Architecture of Systems, LAAS-CNRS, F-31077, Toulouse, France (cescriba@laas.fr).

${ }^{\S}$ E.G.A. BRAVO is with University of Toulouse, Laboratory for Analysis and Architecture of Systems, LAAS-CNRS, F-31077, Toulouse, France (egavinabra@laas.fr).

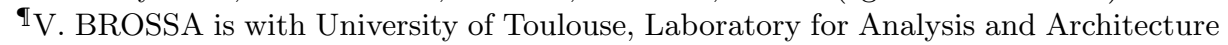
of Systems, LAAS-CNRS, F-31077, Toulouse, France (vbrossa@laas.fr).

$\|$ J.Y. FOURNIOLS is with University of Toulouse, Laboratory for Analysis and Architecture of Systems, LAAS-CNRS, F-31077, Toulouse, France (fourniol@laas.fr).

${ }^{* *} \mathrm{C}$. ROSSI is with University of Toulouse, Laboratory for Analysis and Architecture of Systems, LAAS-CNRS, F-31077, Toulouse, France (rossi@laas.fr).
} 
and other post-impact information to detect that a fall has occurred. These systems can reduce the rescue time, i.e., the time for the first aids arrive to help the person.

One main challenge for next generation FDSs is not to detect when the person fell but to predict an occurring fall (pre-impact) to trigger a protection device, such as an airbag to prevent the injuries and trauma [11,12]. This action requires accurately distinguishing a fall from the activities of daily living (ADL) i.e., walking, sitting. The majority of the existing preimpact fall detection systems are wearable-sensor-based $[11,12]$ and profit from advancements in microelectronics and wireless communication technology. These devices can capture body movement unobtrusively and allow kinematic measurements to be monitored over an extended space.

Most of the articles in the literature use wearable micro-electro-mechanical systems (MEMS), such as integrated inertial measurement units, which typically consist of a triaxial accelerometer and a triaxial gyroscope and use human body kinematics (segment translational measures and rotational measures) as fall detection indicators. Trunk velocity and acceleration [13-16], head acceleration, and upper arm velocity $[16,17]$ can be used to determine the translational measures. Rotational measures can be calculated from the angular rate of the sternum [18], the waist $[12,19]$ and trunk [20,21]. Associated with the sensors, threshold-based algorithms appear to be the simplest algorithms utilized in pre-impact fall detection research because they are computationally efficient. However, setting an appropriate threshold is always difficult, and almost all the current threshold-based techniques face the dilemma with a low threshold leading to false alarms or a high threshold leading to misdetection of falls.

Machine learning (ML) approaches [22-24] are alternatives to thresholdbased techniques and represent approximately $15 \%$ of the literature dedicated to fall-detection. A training period is required to collect the data during non-fall activities and facilitate the features extraction to classify the activity. ML algorithm efficiency depends on the features, dataset, and proportion of training and testing data. In optimized situations, most of the ML algorithms can detect falls with a specificity of $97 \%$ and an accuracy of $98 \%$. Supervised machine learning can also be used to classify the activities $[25,26]$. Deep learning approaches based on adaptive learning facilitate fall detection classifiers, thus improving specificity while using only a single sensor device, such as triaxial accelerometer. However, classic supervised ML approaches remain computationally consuming compared to simple threshold-based approaches, leading to low energy efficiency, which is a key requirement when developing a wearable fall detection system for inflatable airbag deployment. Therefore, despite the promise of ML or deeplearning approaches, we chose herein to implement a FDS based on an efficient threshold algorithm.

Wearable FDS needs to be not only light and small to improve accept- 
ability and usability but also efficient and reliable, i.e, able to discriminate falls from non-fall activities and detect all fall situations. Fall detection accuracy is typically measured by sensitivity and specificity. Sensitivity is determined by the ratio of the number of successfully detected falls to the total number of falls, whereas specificity is determined by the ratio of the number of successfully detected non-fall activities to the total number of non-fall activities. The sensitivities reported in the reviewed studies ranged from 80 to $100 \%$. The sensitivity $(=80 \%)$ in Sabatini et al. [27] was the lowest. The rest of the studies achieve sensitivity above 90\%. Lead time (time interval between when the fall was detected and fall impact) is typically used to assess the efficiency of fall detection. Thus, the longer the lead time is, the better the fall detection performance is. The reported lead time in the literature is from 40 to $750 \mathrm{~ms}$. Shi et al. [12] proposed airbag technology for preventing fall impacts. The inflation time of the airbag was reported to be approximately $130 \mathrm{~ms}$. In order to be effective in avoiding a fall impact, the lead time of fall detection should be longer than $130 \mathrm{~ms}$.

Altogether, the state-of-the-art shows that there are some limitations in the current pre-impact fall detection systems. They often have high computational demand and are expensive and difficult to implement in a real application. In addition, the validity of pre-impact fall models is often limited because the falls used for evaluating fall detection algorithms are often simulated. As a consequence, it is difficult to generalize the currently published results to fall accidents that occur in real life.

This paper presents a wearable pre-impact FDS based on a MEMS accelerometer to capture the body movement (translational and deduced rotational measures from a 3-axis accelerations) coupled with a threshold-based algorithm that also uses the physiological characteristics (height) as input features. The algorithm was evaluated in real conditions using a dataset created on this work with 9 persons and containing 135 falls and 86 ADL. An open online dataset with 38 volunteers (adults and elderly people) was used to validate the method with more realistic cases (ADL followed by a fall). The results demonstrated the ability to detect lateral falls and backwardforward falls with $94.04 \%$ sensitivity and obtained a low percentage of false alarms leading to a $97.67 \%$ specificity. The lead time is $259 \mathrm{~ms}$, which enables the triggering of a protection device such as an airbag. In addition, using only a MEMS accelerometer as a sensor makes the device small, lightweight, and low-cost, which allows the system to be easily implemented in real-time applications for elderly protection.

The paper is organized as follows: Section II first describes the hardware and the pre-impact fall detection algorithm. The evaluation of the systems is presented in Section III. Section IV concludes this research work. 


\section{Materials and methods}

\subsection{Data acquisition}

To facilitate the use of our system, a low-power miniaturized embedded prototype was designed and developed, which is small $(50 \mathrm{~mm} \times 40 \mathrm{~mm} \times$ $16 \mathrm{~mm}$ ) and light $(13 \mathrm{~g})$, meant to be worn on the subject's waist to analyze the acceleration signals and posture. The system is composed of a printed circuit board (PCB) (Fig.1) that contains a single low-power tri-axis MEMS accelerometer (LIS3DH from STMicroelectronics). The sensor is scaled $\pm 8 g$ and oriented with the $\mathrm{x}$-axis for the left; the $\mathrm{y}$-axis downward positive and the z-axis forward positive. To get the best compromise between resolution and consumption, we configured the accelerometer data output in 12-bits resolution with a sampling rate of $400 \mathrm{~Hz}$.

The digital output is transferred through a serial peripheral interface (SPI) to the main component of the system, the nRF52832. This System-onChip (SoC) from Nordic Semiconductor is a low-power consumption SoC [28] integrated with the Bluetooth low energy (BLE) interface, used to communicate with a mobile device (cellphone or tablet). A $2.4 \mathrm{GHz}$ ceramic antenna (Johanson Technology) was integrated on the PCB to increase the BLE signal power. All of the system is powered with a 3V coin cell battery CR2032. The PCB is illustrated in Fig.1.

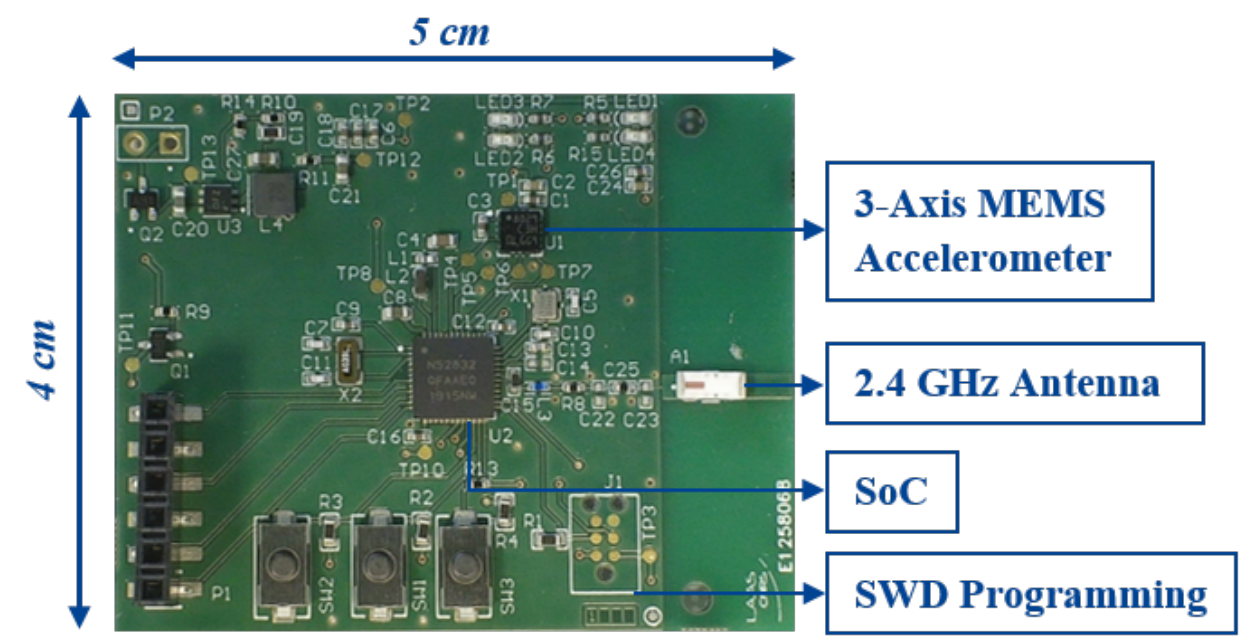

Figure 1: Photo of the prototype of the pre-impact detection system

As this is a first prototype, some components for debugging the system were integrated, such as buttons and connector. For an improved version, its dimensions are meant to be halved. 


\subsection{Pre-impact fall detection algorithm}

This section presents the methodology used to develop the pre-impact fall detection algorithm. Two categories of features that will be employed to detect the fall before the impact: the physiological characteristics (the user height) and sensor data (accelerometer). From the acceleration data, the sum vector magnitude (SVM) of the acceleration is calculated (Eq. (1)), as it gives a first view of the impact time and the particular shape of the signal. Where $a_{x}(t), a_{y}(t)$ and $a_{z}(t)$ are the components of the acceleration along each axis, expressed in units of $g\left(9.81 \mathrm{~m} / \mathrm{s}^{2}\right)$.

$$
|\vec{a}(t)|=\sqrt{a_{x}^{2}(t)+a_{y}^{2}(t)+a_{z}^{2}(t)}
$$

The tilt angle of the y-axis along with the gravity ( $\beta$ in rad) is estimated using the Eq. (2). Notably, during dynamic movements, the reliability of this approach is reduced. The analysis of the angles for classifying the activity is complex and difficult to predict because each subject has a different behavioral posture.

$$
\beta=\cos ^{-1}\left(\frac{a_{y}(t)}{\sqrt{a_{x}^{2}(t)+a_{y}^{2}(t)+a_{z}^{2}(t)}}\right)
$$

However, this system uses the tilt angle of y-axis as input for our equation system to predict the loss of balance. The algorithm was elaborated from the classical kinematic Torricelli's Equation (Eq. (3)) which describes a linear movement with constant acceleration along an axis. Where $v$ and $v_{0}$ represents the final and the initial velocities $(\mathrm{m} / \mathrm{s})$ of the body, respectively. The $\Delta x$ is the distance traveled in meters and $a$ is the acceleration $\left(\mathrm{m} / \mathrm{s}^{2}\right)$.

$$
v^{2}=v_{0}^{2} \pm 2 a \Delta x
$$

Considering that the fall has an angular movement, during a static fall, the person performs a rotational movement around the axis that can be approximated between the point of contact with the ground (the feet) and the center of mass. Torricelli's Equation (Eq. (4)) can also be presented to describe a rotational kinematic movement:

$$
\omega^{2}=\omega_{0}^{2} \pm 2 \alpha \Delta \theta
$$

Where $\Delta \theta$ is the rotational angle expressed in radians, $\alpha$ is the angular acceleration $\left(\mathrm{rad} / \mathrm{s}^{2}\right)$. The final and initial angular velocities $\left(\omega\right.$ and $\omega_{0}$, expressed in $\mathrm{rad} / \mathrm{s}$ ) during a fall were modeled in [29,30]. In [29], a lateral fall is defined (Eq. 5), where $l$ is the distance in meters between the waist and the ground and $\varphi$ is the tilt angle (rad) when falling. They considered that the movement starts from a position in which the tilt angle is 0 rad. 


$$
\omega^{2}=\frac{3 g(1-\cos \varphi)}{l}
$$

Mastorakis et al. [30] approximated the fall of a person as a rod falling with uniform mass distribution. For this model, it is considered that the only acceleration on the movement is the gravity $g$, with the assumption that the potential and kinetic energies are preserved. The model indicates the velocity is proportional to the height, a taller person takes longer to fall, and on the impact moment, has a higher velocity. However, the acceleration behaviour is independent of height.

We use the concept presented in [30] to define the angular velocity in Eq. (6).

$$
\omega_{n}=\sqrt{\omega_{n-1}^{2}+\frac{3 g\left(\cos \beta_{n-1}-\cos \beta_{n}\right)}{h_{1}}}
$$

Where $h_{1}$ is the distance in centimeters between the PCB position and the ground, i.e the height from the navel to the ground (user's feet). Eq. (4) and Eq. (6) demonstrated that the angular displacement $\Delta s(\mathrm{~cm})$ of a person between two acquisition $n-1$ and $n$, can be defined as shown in Eq. (7).

$$
\Delta s=\frac{3}{2} h_{1} \times\left[\cos \left(\beta_{n-1}\right)-\cos \left(\beta_{n}\right)\right]
$$

To obtain the total displacement on a time interval $t$.

$$
S=\sum \Delta s=\sum_{1}^{n} \frac{3}{2} h_{1} \times\left[\cos \left(\beta_{n-1}\right)-\cos \left(\beta_{n}\right)\right]
$$

The third important feature of the algorithm is the distance $d$ normal to the ground. Assume that in a static condition, height $h_{1}$ is equal to $d$. The distance is variable according to the movement, and it can be periodic as well as $S$ for some ADL activities (e.g. walk).

The Eq. (9) shows that the $d_{n}$ behaviour depends on the tilt angle between the y-axis and gravity vector, as well as on the user's physiological characteristics.

$$
d_{n}=h_{1} \cos \beta_{n}
$$

To reduce the false-positive cases, a loss of balance condition was defined as the moment when the displacement of the body $(S)$ is greater than the normal distance from the ground $\left(d_{n}\right)$. Assuming that that $S<d_{n}$, the person is in a safe region, and this was defined as a "Balanced boundary circle", in which the chance that a fall occurs is minimal. In contrast, when the person is out of the circle, the algorithm analyses the acceleration to identify an impending fall condition to trigger the protection system. 


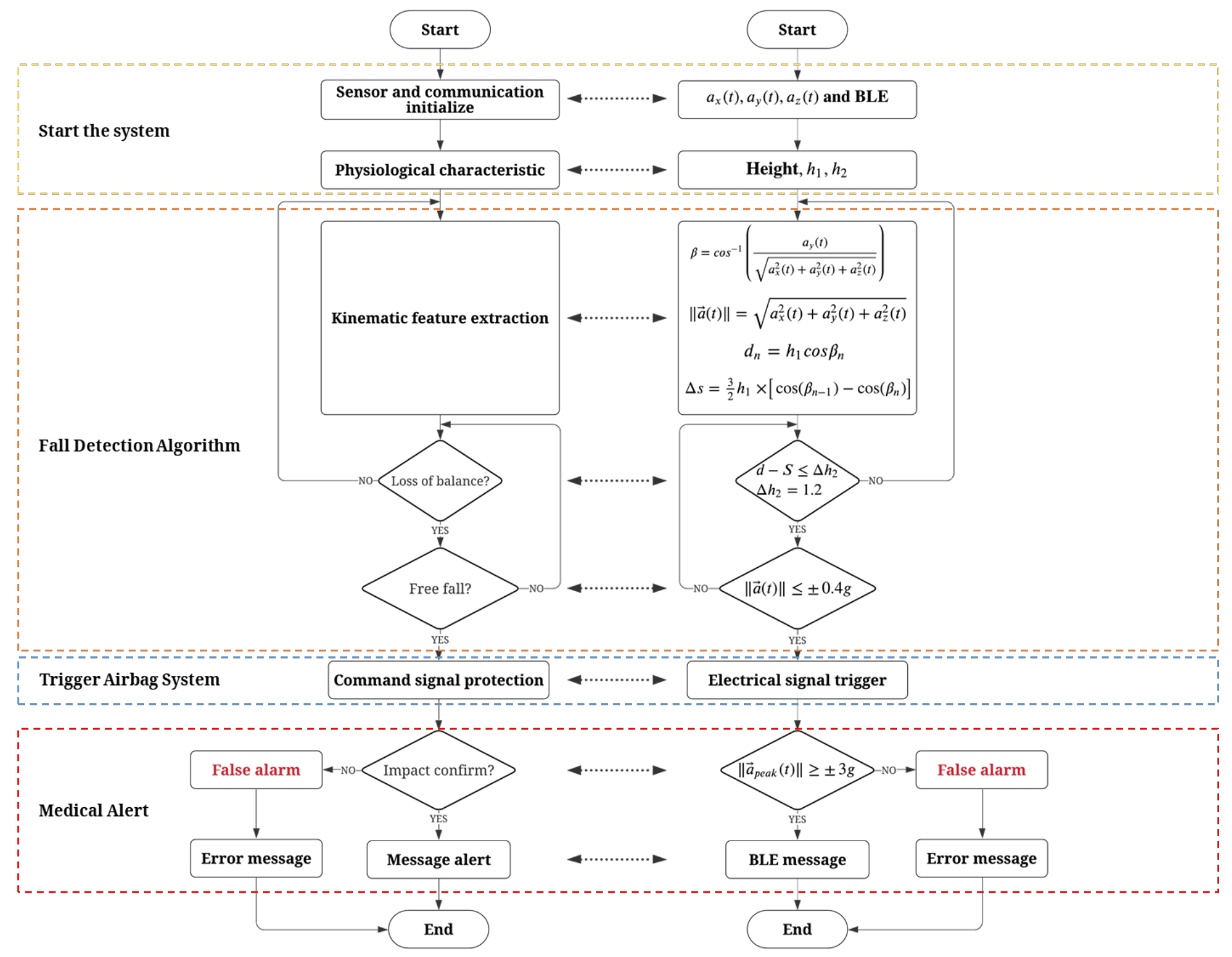

Figure 2: Flow chart of the system algorithm - Left side shows the logic of the flow and right side represent the equations and decisions of the algorithm. 
It is important to know that an ADL activity has a stage that can be misinterpreted as a lack of balance, the swing phase $[31,32]$. The condition of the balanced boundary circle is not enough to reduce false alarms. Alternatively, the combination of this condition with the analysis of the body acceleration makes the algorithm more robust.

The entire algorithm is presented in Fig. 2. The left side represents the logical flow to detect the fall before the impact, and on the right, the equations used on each step. The algorithm is divided into three main steps: the fall detection algorithm, user protection with an airbag system and a message alert for first aid. The present work focuses on the second step, i.e., the threshold-based fall detection algorithm.

To improve the algorithm and the lead time response, a second physiological characteristic was used, $h_{2}$ (distance in centimeters between the head and the PCB), as a threshold to identify a near loss of balance situation. This choice is based on the fact the height influences the balance.

The aim of the detection is to identify the fall as soon as possible with fewer false alarms. The algorithm uses an empirical threshold (1.2 factor of $h_{2}$ ) to predict the lack of balance faster and a $0.4 \mathrm{~g}$ threshold of the SVM to confirm the user is falling. The choice of the threshold values is described in the section III.

A customizable threshold-based pre-impact fall detection algorithm was developed to be used for protection to avoid injury from falls. The algorithm is adapted for each person according to their height. The critical goal of our system is to detect the fall before the impact and determine the instant of an impending fall, to predict a condition of the loss of balance. 


\subsection{Tuning the Fall Detection Algorithm threshold}

We created a dataset performing the tests using two prototypes worn on the volunteers. The PCBs were placed on a belt to guarantee that both have the same distance to the ground.

The subjects wore one prototype on the back and another one in front of the body, and the program analysed the data in real-time and calculated the accelerations data, taking into account the PCB position influence. The nine volunteers ( 2 females and 7 males) were all in good health, and they did not present any physical condition that could interfere with the tests (height average $174.11 \pm 6.98 \mathrm{~cm}$; weight average $63.57 \pm 7.91 \mathrm{~kg}$ ). The volunteers gave their consent to participle in this study.

We asked the subjects to realize several falls in all the directions and 5 ADL (walk, run, sit, stand up and bend to pick an object). Each activity is repeated five times. The sensor data is sampled at $400 \mathrm{~Hz}$ then sent through BLE link to a computer to be recorded. The subjects were asked to relax their falls, try to keep their knees unbent and not use their hands to protect them from the impact, simulating a real fall. A total of 135 falls and 86 ADLs were recorded and used to develop and test our algorithm. We analysed 10 seconds of the ADL and 5 seconds of the falls. 


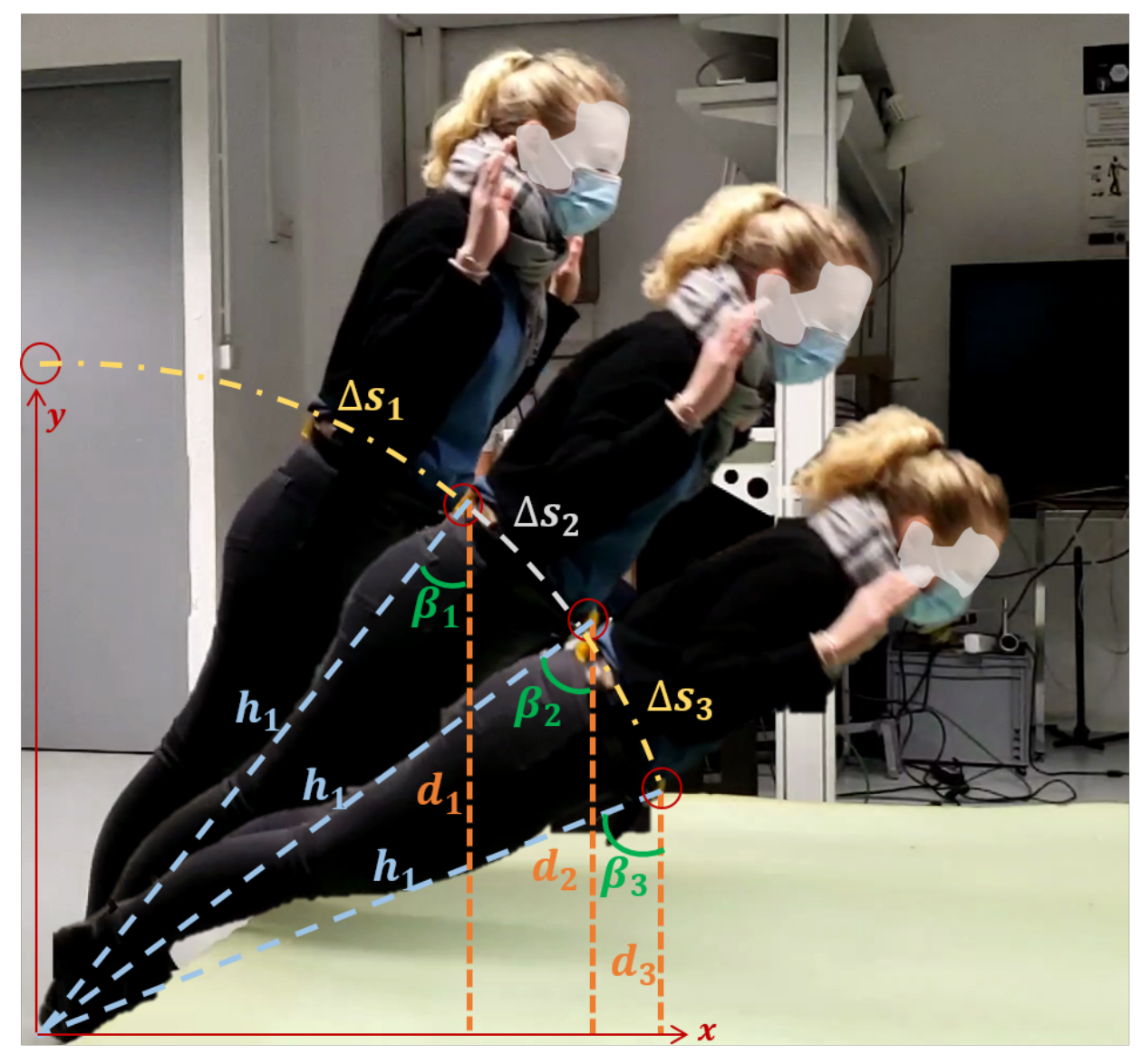

Figure 3: Photo of a Forward Fall simulation

The physiological features used in the algorithm are represented in Fig.3. The distance $d$ decreases during a fall situation, while the angle $(\beta)$ value tends to $90^{\circ}$.

The data of the prototype on the back was less noisy, but based on the volunteers reports about the comfort and for the ergonomic reasons, we chose to use the data from the PCB placed on the front, also because this PCB was near the mass center. The raw data of different ADL and a Forward Fall are shown in Fig.4. 

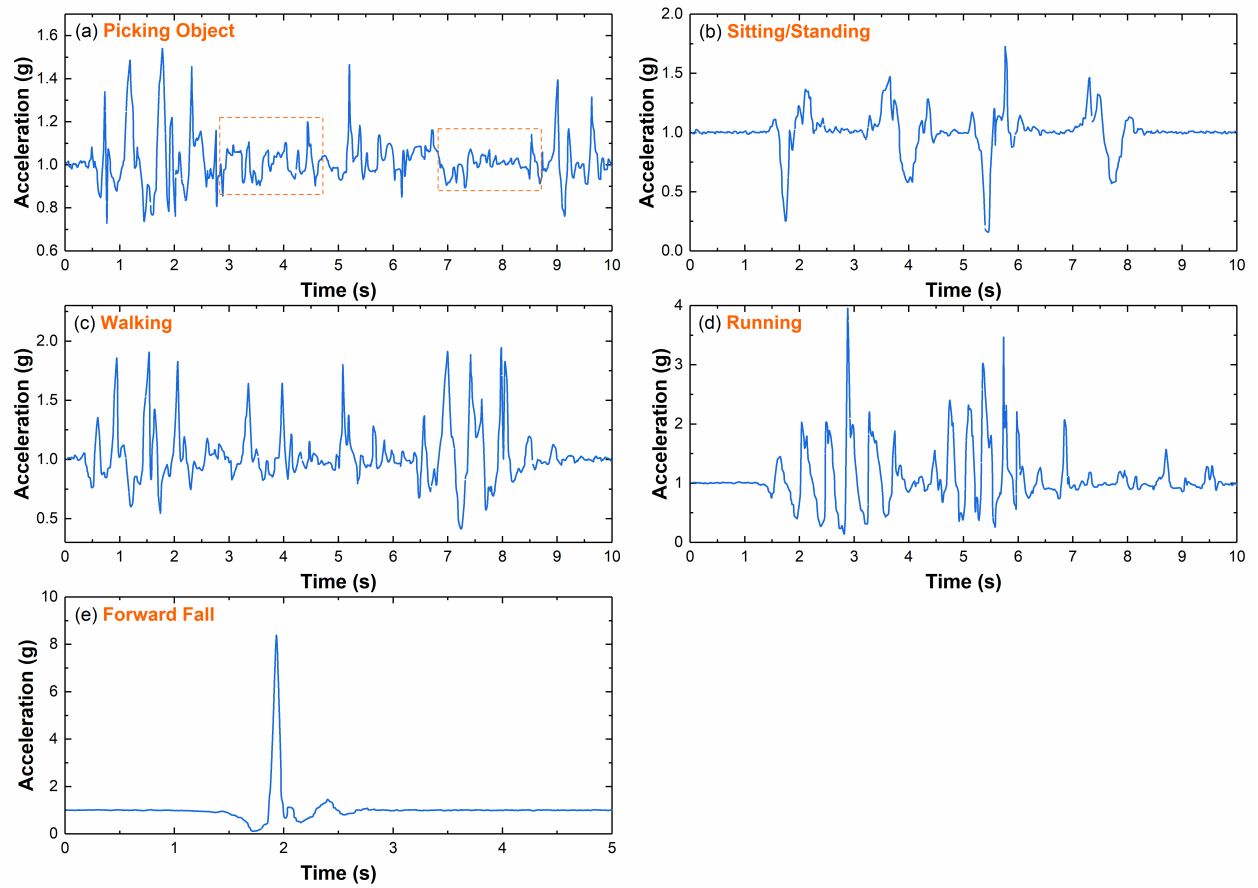

Figure 4: Sum Vector Magnitude of ADL (a,b,c,d) and a Forward Fall (e)

The sum vector magnitude of the acceleration has the same behaviour in all the directions [33], i.e., the SVM feature can not graphically differentiate the direction of the fall. We also observed that the lateral falls had the lowest peak values, as well as the most distant free-fall conditions (minimum value), due to the fact that the subjects bent their knees to protect themselves.

Although the signature of a fall is recognized graphically, the dynamics of the ADL can be interpreted as a near-fall condition. A fall is characterized by a decay of acceleration followed by a sudden change in slope and a peak, behaviour similar to some ADLs.

The SVM is an important feature to distinguish these activities, mainly when analyzing the maximum value. Summarized averages results of each activity, are given in Table 1 . 
Table 1: SVM values of each activity

\begin{tabular}{|c|c|c|}
\hline Activity & SVM Min & SVM Max \\
\hline Sitting & $0.2 \mathrm{~g}$ & $1.8 \mathrm{~g}$ \\
Standing & $0.4 \mathrm{~g}$ & $1.6 \mathrm{~g}$ \\
Picking Object & $0.8 \mathrm{~g}$ & $1.2 \mathrm{~g}$ \\
Walk & $0.5 \mathrm{~g}$ & $2.0 \mathrm{~g}$ \\
Run & $0.35 \mathrm{~g}$ & $3.0 \mathrm{~g}$ \\
Backward Fall & $0.09 \mathrm{~g}$ & $9.2 \mathrm{~g}$ \\
Forward Fall & $0.085 \mathrm{~g}$ & $7.8 \mathrm{~g}$ \\
Rightward Fall & $0.18 \mathrm{~g}$ & $6.2 \mathrm{~g}$ \\
Leftward Fall & $0.13 \mathrm{~g}$ & $6.2 \mathrm{~g}$ \\
\hline
\end{tabular}

The maximum magnitude is due to the impact; this is the reason why the peak value of the falls is higher than the ADL. However, this information is not valid for our purpose, which is to develop a pre-impact fall detection system to protect the person before reaching the ground. The final version of the algorithm was transcript in $C$ to program the System-on-Chip (NRF52832). An average consumption of $5.91 \mathrm{~mA}$ was measured.

\subsection{Performance Assessment}

The results are analysed based on statistical parameters, such as sensitivity (SE), specificity (SP), accuracy (AC) which are defined by concepts of true positives (TP), true negatives (TN), false positives (FP), and false negatives $(\mathrm{FN})$ as follows:

- The sensitivity (SE) is the ability of the system to identify falls

$$
S E=\frac{T P}{T P+F N}
$$

- The specificity (SP) is the capability to correctly detect ADL as ADL (measure the number of false alarms)

$$
S P=\frac{T N}{T N+F P}
$$

- The accuracy (AC) is the ability of the system to discern Falls and ADLs

$$
A C=\frac{S E+S P}{2}
$$

- Lead time (LD) is the time left for the system to activate the protection mechanisms until the moment of impact. 
The terms true and negative are used to refer to the presence or absence of the condition of a fall. True positives are the main instances of interest whereas true negatives are all other instances. As mentioned before, to classify a person within the balanced boundary circle, the ground distance (d) is subtracted from the total angular displacement $(S)$ during an interval $t$. The $0.4 g$ was chosen as the free-fall condition threshold because, as noted in the Table 1, the minimum SVM of most ADL is higher than $0.4 g$ (except for the sitting activity $0.2 g$ ). However, for this ADL, the body tilt during the activity is too small to exceed the lack of balance threshold. A fall is detected when both conditions are confirmed.

\section{Results and discussion}

In Fig 5, the periodic behaviour of a walking activity is illustrated. The algorithm predicts a possible loss of balance and starts to analyze the acceleration. A period of a loss of balance (the person is out of the Balanced boundary circle) is observed at time $t \cong 2.5 \mathrm{~s}$, but due the acceleration still greater than $0.4 \mathrm{~g}$, this is classified as an ADL.

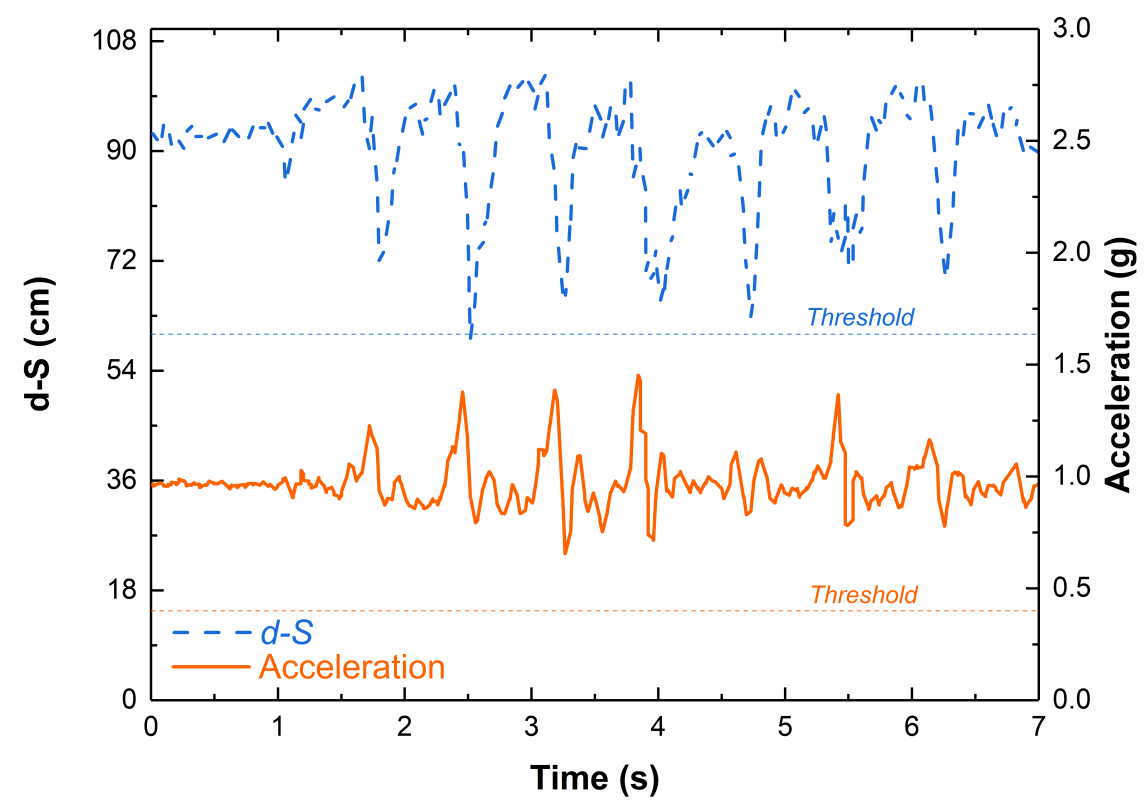

Figure 5: Data obtained from a Walk activity

Alternatively, when a fall is happening, the SVM and the $d-S$ curve have the same sense of variation, followed by a sudden peak change, as shown in Fig. 6. The negative value is explained because the angular displacement 
$S$ is approximated $h_{1} \frac{\pi}{2}$ when $d_{n}=0$.

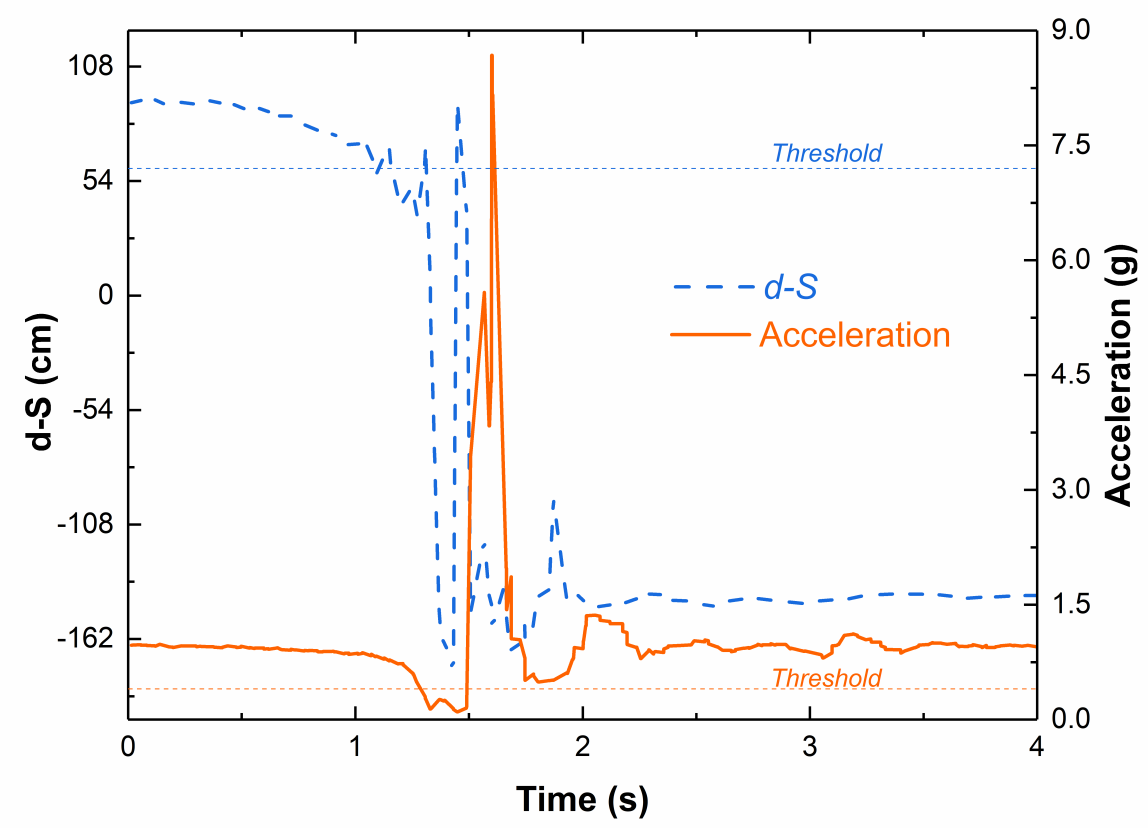

Figure 6: Data obtained from a Forward Fall

The threshold-based algorithm correctly detected 125 falls and 71 ADL, which corresponds to $92.59 \%, 83.72 \%$ and $88.16 \%$ of sensitivity, specificity and accuracy, respectively. The lower specificity is justified by the fact the ADL recorded was made by younger persons, which are faster than elderly persons. As mentioned before (section 2.B), for dynamic movements the algorithm presents a low accuracy but is still reliable. The lead time average is $189.41 \mathrm{~ms}$, which is long enough to ensure an individual's protection.

We observed that the balanced boundary circle threshold has to be customizable for each person. The algorithm performance is reduced by applying a fixed and constant threshold. If we use the average of $h_{2}$, we achieved $86 \%$ of accuracy with a high value of false alarms (20\%).

To optimize the customizable threshold to improve the robustness of the algorithm, the threshold was varied according to the user height. Fig. 7 illustrates that the optimal threshold is $1.2 h_{2}$, the $\mathrm{x}$-axis values increase from the left to the right. 


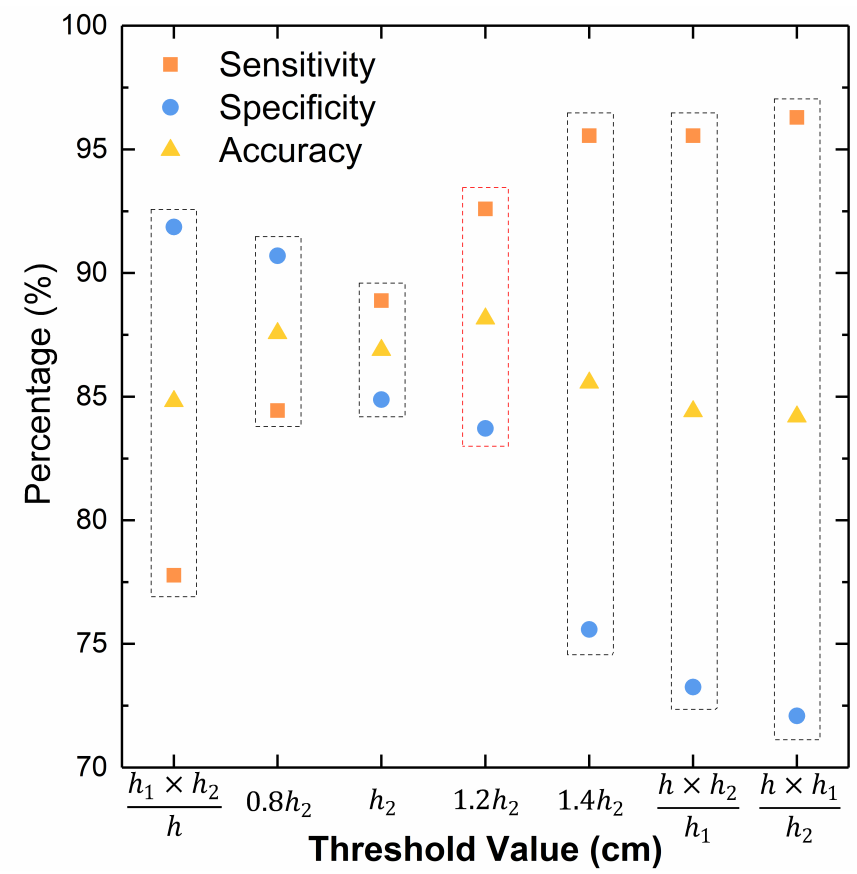

Figure 7: Balanced Boundary circle threshold variation - values increase from the left to right

With a higher threshold value, the number of false positives increases because the fall prediction is early, so any abnormal acceleration can be misinterpreted as a fall. With a lower threshold value, the prediction is too late for the system to deploy the protection system.

To further characterize the performance of our algorithm, an online public database was used, named SisFall [34]. The SisFall database contains 19 types of ADL and 15 types of falls. The activities were recorded from 38 volunteers, 23 adults and 15 elderly persons (from 60 to 75 years old). Only one elderly participant executed the falls. Some ADL could not be realized for medical reasons of the volunteers. In total, the dataset has 1798 falls and 2706 ADL recorded from three sensors (2 accelerometers and 1 gyroscope) worn on the waist. 


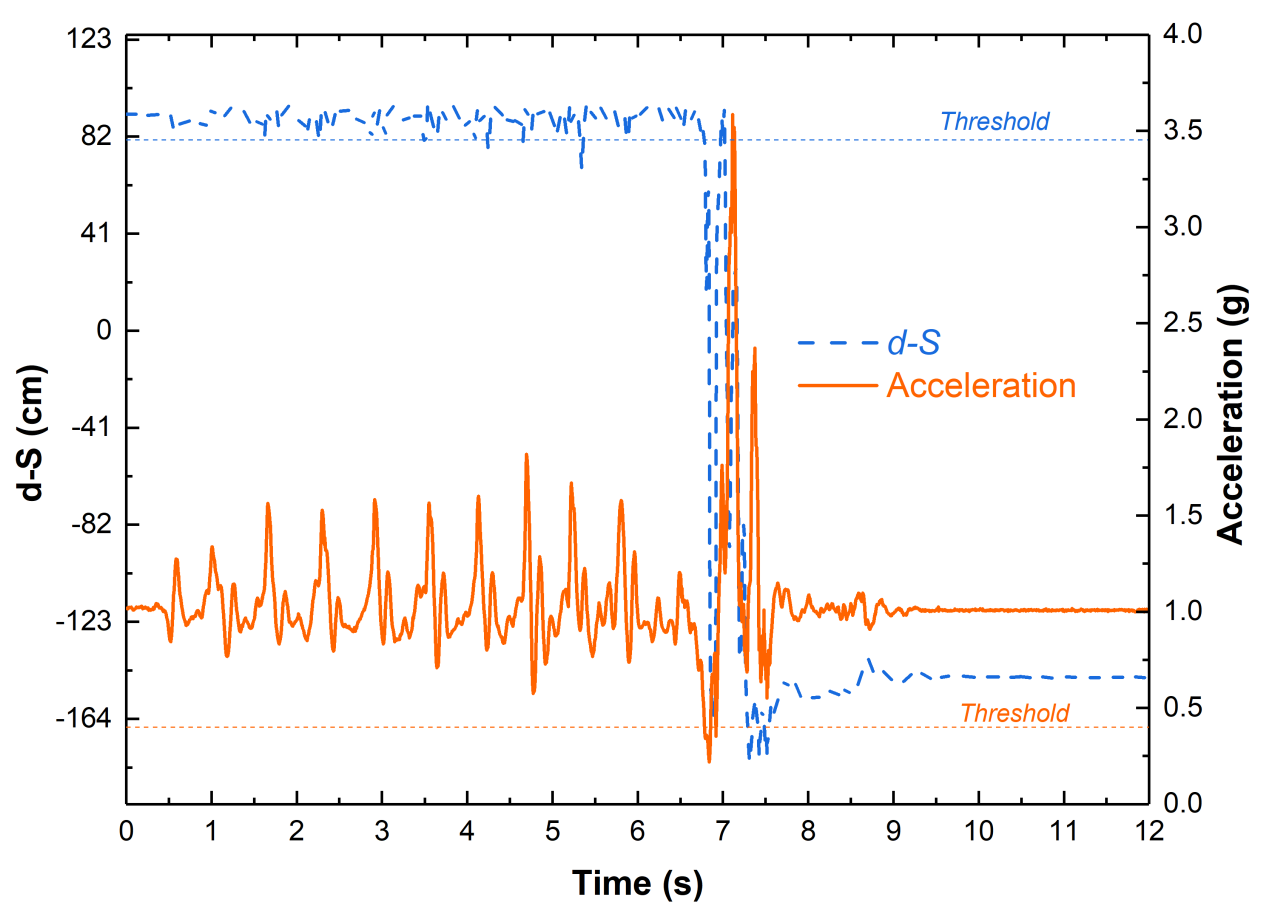

Figure 8: Data from Sisfall - Lateral fall while walking caused by a slip

As the purpose of this study is to develop an algorithm to protect elderly persons, we did not take into account fast activities to validate our method. Thus, only 10 ADL from 19 were chosen. We used 1672 ADL and achieved $97.67 \%$ of specificity. We confirm the fact that the ADL execution from our database was faster than a normal activity.

To evaluate the sensitivity, we chose the fall while walking in all directions and a fall while sitting to analyze the capability to detect a fall when the ADL is a like-fall activity. With 537 falls the algorithm archive $94.04 \%$ of sensitivity with an average lead time of $259 \mathrm{~ms}$. The accuracy was of $95.86 \%$ for this test.

In Fig. 8 presents a more realistic scenario corresponding to a fall occurring during an ADL, i.e, walking. A fall is detected when both threshold are achieved. During the ADL activity, when the loss of balance is less common, the algorithm do not calculate the SVM of the acceleration, reducing the consumption.

Some recent studies present good accuracy results (Table 2), although with fewer cases analyses and a reduced number of volunteers. Wu et al. [35] focused in the forward and backward fall detection using two IMUs placed on the user (waist and right thigh). The dataset used was formed by 15 volunteers. Shi et al. [22] used an acceloremeter and a gyroscope placed at the waist to analyze the data from 9 volunteers to detect the fall in all the directions. Compared to these studies, this work considered a greater 
diversity of cases, 10 ADL and 5 falls, performed by more people (38 from Sisfall).

Table 2: Performance Comparison

\begin{tabular}{|c|c|c|c|}
\hline & Wu et al. [35] & Shi et al. [22] & This Study \\
\hline Sensitivity & $95.5 \%$ & $94.52 \%$ & $94.04 \%$ \\
Specificity & $97.3 \%$ & $96.52 \%$ & $97.67 \%$ \\
Accuracy & $96.4 \%$ & $95.55 \%$ & $95.86 \%$ \\
Lead time & 346 ms- $404 \mathrm{~ms}$ & Not specified & $259 \mathrm{~ms}$ \\
\hline
\end{tabular}

\section{Conclusions}

In this study, a pre-fall detection system using a MEMS 3D accelerometer (LIS3DH), associated with SoC NRF52832 and $2.4 \mathrm{GHz}$ antenna was developed. The hardware and algorithm were designed to get a low power consumption, very high sensitivity and specificity, and early pre-fall detection to trigger safety devices such as a wearable inflatable airbag.

The algorithm is simply based on the following hypothesis: "A region defined as balanced boundary circle, based on the user's height, is characterized by the fact that the chance that an actual fall happening is minimal. When an activity is classified out this circle, an acceleration analysis is performed to determine an impending fall condition".

Importantly, this threshold-based algorithm not only calculates the translational and deduced rotational measures from a 3-axis accelerometer, but it also employs the subject's height to reduce the number of false alarms compared to current fall detection systems published in the literature. The entire pre-fall system was validated with 9 young healthy volunteers performing both normal ADL and fall activities and using 10 ADL and 5 falls from the public SisFall dataset.

The results show that the system can detect front, back and side falls without a priori knowledge, consuming $5.83 \mathrm{~mA}$ during the sensor acquisition and $6.35 \mathrm{~mA}$ in calculation mode, i.e., ADL with or without fall. The results also show that falls can be detected with an average lead time of 259 $\mathrm{ms}$ before the impact occurs, with few false alarms (97.7\% specificity) and a sensitivity of $92.6 \%$. This is a very good lead-time achieved thus far in pre-impact fall detection, permitting the integration of our detection system into a wearable inflatable airbag.

The integration of the entire miniaturized final system $(5 \mathrm{~g})$ with the optimized pre-impact fall detection system $(3 \mathrm{~cm} \times 3 \mathrm{~cm})$, a trigger circuit $(2 \mathrm{~cm} \times 2 \mathrm{~cm})$ and a gas generator $(4 \mathrm{~cm} \times 3 \mathrm{~cm})$ is currently underway. 


\section{Acknowledgment}

The authors acknowledge support from the European Research Council (H2020 Excellent Science) Researcher Award (grant 832889 - PyroSafe) and the Occitanie Region / European Union for their FEDER support (THERMIE grant). The authors also thank all the volunteers who had participated in our experiment.

\section{References}

[1] M. C. Nevitt, S. R. Cummings, and E. S. Hudes, "Risk Factors for Injurious Falls: a Prospective Study," Journal of Gerontology, vol. 46, pp. M164-M170, Sept. 1991.

[2] R. W. Sattin, "Falls Among Older Persons: A Public Health Perspective," p. 20.

[3] M. R. Kosorok, G. S. Omenn, P. Diehr, T. D. Koepsell, and D. L. Patrick, "Restricted activity days among older adults.," American Journal of Public Health, vol. 82, pp. 1263-1267, Sept. 1992.

[4] M. E. Tinetti, J. Doucette, E. Claus, and R. Marottoli, "Risk Factors for Serious Injury During Falls by Older Persons in the Community," Journal of the American Geriatrics Society, vol. 43, pp. 1214-1221, Nov. 1995.

[5] R. Cuevas-Trisan, "Balance Problems and Fall Risks in the Elderly," Physical Medicine and Rehabilitation Clinics of North America, vol. 28, pp. 727-737, Nov. 2017.

[6] W. H. Organization, ed., WHO global report on falls prevention in older age. Geneva, Switzerland: World Health Organization, 2008. OCLC: ocn226291980.

[7] Y. Li, G. Chen, Y. Shen, Y. Zhu, and Z. Cheng, "Accelerometer-based fall detection sensor system for the elderly," in 2012 IEEE 2nd International Conference on Cloud Computing and Intelligence Systems, (Hangzhou, China), pp. 1216-1220, IEEE, Oct. 2012.

[8] J. Santiago, E. Cotto, L. G. Jaimes, and I. Vergara-Laurens, "Fall detection system for the elderly," in 2017 IEEE 7th Annual Computing and Communication Workshop and Conference (CCWC), (Las Vegas, NV, USA), pp. 1-4, IEEE, Jan. 2017.

[9] M. Vallejo, C. V. Isaza, and J. D. Lopez, "Artificial Neural Networks as an alternative to traditional fall detection methods," in 2013 35th 
Annual International Conference of the IEEE Engineering in Medicine and Biology Society (EMBC), (Osaka), pp. 1648-1651, IEEE, July 2013.

[10] J. Dai, "PerFallD: A Pervasive Fall Detection System Using Mobile Phones," p. 6.

[11] T. Tamura, M. Uchida, and O. Tanaka, "A Wearable Airbag to Prevent Fall Injuries," IEEE TRANSACTIONS ON INFORMATION TECHNOLOGY IN BIOMEDICINE, vol. 13, no. 6, p. 5, 2009.

[12] G. Shi, C. S. Chan, W. J. Li, K.-S. Leung, Y. Zou, and Y. Jin, "Mobile Human Airbag System for Fall Protection Using MEMS Sensors and Embedded SVM Classifier," IEEE Sensors Journal, vol. 9, pp. 495-503, May 2009.

[13] A. K. Bourke, K. J. O'Donovan, J. Nelson, and G. M. OLaighin, "Falldetection through vertical velocity thresholding using a tri-axial accelerometer characterized using an optical motion-capture system," in 2008 30th Annual International Conference of the IEEE Engineering in Medicine and Biology Society, (Vancouver, BC), pp. 2832-2835, IEEE, Aug. 2008.

[14] A. Bourke and G. Lyons, "A threshold-based fall-detection algorithm using a bi-axial gyroscope sensor," Medical Engineering $\&$ Physics, vol. 30, pp. 84-90, Jan. 2008.

[15] X. Hu and X. Qu, "Detecting falls using a fall indicator defined by a linear combination of kinematic measures," Safety Science, vol. 72, pp. 315-318, Feb. 2015.

[16] X. Hu and X. Qu, "An individual-specific fall detection model based on the statistical process control chart," Safety Science, vol. 64, pp. 13-21, Apr. 2014.

[17] U. Lindemann, A. Hock, M. Stuber, W. Keck, and C. Becker, "Evaluation of a fall detector based on accelerometers: A pilot study," Medical ES Biological Engineering $\&$ Computing, vol. 43, pp. 548-551, Oct. 2005.

[18] M. Nyan, F. Tay, A. Tan, and K. Seah, "Distinguishing fall activities from normal activities by angular rate characteristics and high-speed camera characterization," Medical Engineering \& Physics, vol. 28, pp. 842-849, Oct. 2006.

[19] M. Nyan, F. E. Tay, and E. Murugasu, "A wearable system for preimpact fall detection," Journal of Biomechanics, vol. 41, pp. 3475-3481, Dec. 2008. 
[20] Jian Liu and T. E. Lockhart, "Development and Evaluation of a Priorto-Impact Fall Event Detection Algorithm," IEEE Transactions on Biomedical Engineering, vol. 61, pp. 2135-2140, July 2014.

[21] J. Liu and T. E. Lockhart, "Automatic individual calibration in fall detection - an integrative ambulatory measurement framework," Computer Methods in Biomechanics and Biomedical Engineering, vol. 16, pp. 504-510, May 2013.

[22] J. Shi, D. Chen, and M. Wang, "Pre-Impact Fall Detection with CNNBased Class Activation Mapping Method," Sensors, vol. 20, p. 4750, Aug. 2020.

[23] T. H. Kim, A. Choi, H. M. Heo, K. Kim, K. Lee, and J. H. Mun, "Machine learning-based pre-impact fall detection model to discriminate various types of fall," Journal of biomechanical engineering, vol. 141, no. $8,2019$.

[24] M. Luštrek and B. Kaluža, "Fall detection and activity recognition with machine learning," Informatica, vol. 33, no. 2, 2009.

[25] S. Chernbumroong, S. Cang, A. Atkins, and H. Yu, "Elderly activities recognition and classification for applications in assisted living," Expert Systems with Applications, vol. 40, no. 5, pp. 1662-1674, 2013.

[26] R. Jain, V. B. Semwal, and P. Kaushik, "Deep ensemble learning approach for lower extremity activities recognition using wearable sensors," Expert Systems, p. e12743, 2021.

[27] A. M. Sabatini, G. Ligorio, A. Mannini, V. Genovese, and L. Pinna, "Prior-to- and Post-Impact Fall Detection Using Inertial and Barometric Altimeter Measurements," IEEE Transactions on Neural Systems and Rehabilitation Engineering, vol. 24, pp. 774-783, July 2016.

[28] D. Oletic and V. Bilas, "System-Level Power Consumption Analysis of the Wearable Asthmatic Wheeze Quantification," Journal of Sensors, vol. 2018, pp. 1-18, 2018.

[29] G. Shi, C.-s. Chan, Y. Luo, G. Zhang, W. J. Li, P. H. W. Leong, and K.-s. Leung, "Development of a Human Airbag System for Fall Protection Using MEMS Motion Sensing Technology," in 2006 IEEE/RSJ International Conference on Intelligent Robots and Systems, (Beijing), pp. 4405-4410, IEEE, Oct. 2006.

[30] G. Mastorakis, T. Ellis, and D. Makris, "Fall detection without people: A simulation approach tackling video data scarcity," Expert Systems with Applications, vol. 112, pp. 125-137, Dec. 2018. 
[31] V. B. Semwal, S. A. Katiyar, R. Chakraborty, and G. C. Nandi, "Biologically-inspired push recovery capable bipedal locomotion modeling through hybrid automata," Robotics and Autonomous Systems, vol. 70, pp. 181-190, 2015.

[32] P. Patil, K. S. Kumar, N. Gaud, and V. B. Semwal, "Clinical human gait classification: extreme learning machine approach," in 2019 1st international conference on advances in science, engineering and robotics technology (ICASERT), pp. 1-6, IEEE, 2019.

[33] F. Bagala, C. Becker, A. Cappello, L. Chiari, K. Aminian, J. M. Hausdorff, W. Zijlstra, and J. Klenk, "Evaluation of accelerometer-based fall detection algorithms on real-world falls," PloS one, vol. 7, no. 5, p. e37062, 2012.

[34] A. Sucerquia, J. López, and J. Vargas-Bonilla, "SisFall: A Fall and Movement Dataset," Sensors, vol. 17, p. 198, Jan. 2017.

[35] Y. Wu, Y. Su, R. Feng, N. Yu, and X. Zang, "Wearable-sensor-based pre-impact fall detection system with a hierarchical classifier," Measurement, vol. 140, pp. 283-292, July 2019. 